\title{
Intermolecular Coupling of Alkenes to Heterocycles via Rhodium Catalyzed C-H Bond Activation
}

\author{
Kian L. Tan, Robert G. Bergman*, and Jonathan A. Ellman* \\ Department of Chemistry, University of California and Division of Chemical Sciences, \\ Lawrence Berkeley National Laboratory \\ Berkeley, CA 94720
}

\section{Supporting Information}

I. Spectroscopic and Analytical Data

Page \#

II. Additive Table

(a) General Procedure for Additive Screen

S-10

(b) Table S-1. Table of Additives Screened

S-10

III. Structural Data for 3 .

S-11

(a) Figure S-3. ORTEP Diagram of 9

S-11 


\section{Spectroscopic and Analytical Data General Experimental}

Unless otherwise noted, all reagents were obtained from commercial suppliers and used without further purification. All solvents were distilled under nitrogen from the following drying agents immediately prior to use: ether and tetrahydrofuran (THF) from sodium/benzophenone ketyl, and dichloromethane $\left(\mathrm{CH}_{2} \mathrm{Cl}_{2}\right)$ from calcium hydride. All reagent solutions were handled under an inert nitrogen atmosphere using syringe and cannula techniques. All reactions unless otherwise noted were carried out in flame or oven-dried glassware under an inert nitrogen atmosphere. Reaction progress was monitored with thin layer chromatography on Merck $60 \mathrm{~F}_{254} 0.25$ micron silica plates. Unless otherwise specified, extracts were dried over $\mathrm{Na}_{2} \mathrm{SO}_{4}$ and solvents were removed with a rotary evaporator. Flash column chromatography was carried out using Merck 60 230-400 mesh silica gel. All infrared (IR) spectra were recorded on a Mattson Galaxy 3000 Fourier Transform spectrometer on thin film on $\mathrm{NaCl}$ plates or $\mathrm{KBr}$ pellets and only partial data are listed. ${ }^{1} \mathrm{H}$ NMR spectra were obtained on a Bruker AMX-400, AM-500 FT, or AMX-300 spectrometers. Unless otherwise noted, all NMR spectra were obtained in $\mathrm{CDCl}_{3}$; chemical shifts are reported in ppm relative to internal standard $\mathrm{CDCl}_{3}$. Coupling constants are reported in Hz. Elemental analyses were performed by M-H-W Laboratories (Phoenix, AZ) and the University of California, Berkeley elemental analysis facility. Mass Spectrometry was performed by the University of California, Berkeley mass spectrometry facility on group instruments. Analytical data and synthetic protocols have been reported for the following compounds in the literature: compound $\mathbf{1}$, $\left[\mathrm{RhCl}(\mathrm{coe})_{2}\right]_{2}{ }^{\mathrm{ii}}$, tert-butyldiphenylsilyl 5-hexen-1-yl ether, ${ }^{\mathrm{iii}}$ and 4, 4, 5, 5-tetramethyl-2vinyl-[1,3] dioxolane. ${ }^{\text {iv }}$

\section{General procedure coupling of heterocycles to alkenes}

Method A. In air to an oven dried vessel with a Kontes stopper was added $\left[\mathrm{RhCl}(\mathrm{coe})_{2}\right]_{2}(5 \mathrm{~mol} \% \mathrm{Rh})(\mathrm{coe}=$ cis-cyclooctene $)$, tricyclohexylphosphine $(7.5 \mathrm{~mol} \%)$, 
lutidinium chloride (5 mol\%), and the heterocycle. The vessel was then placed under nitrogen. Under a positive pressure of nitrogen, THF (diluted to $0.1 \mathrm{M}$ in heterocycle) and the alkene ( 5 equiv) were added to the vessel. The vessel was closed with the Kontes stopper and then was subjected to three successive freeze pump thaw cycles. The vessel was then heated for $15 \mathrm{~h}$ at $150{ }^{\circ} \mathrm{C}$. The reaction mixture was then dry loaded onto $\mathrm{SiO}_{2}$ and purifed by flash chromatography using EtOAc/hexanes.

Method B. In air to an oven dried vessel with a Kontes stopper was added $\left[\mathrm{RhCl}(\mathrm{coe})_{2}\right]_{2}(5 \mathrm{~mol} \% \mathrm{Rh})(\mathrm{coe}=$ cis-cyclooctene $)$, tricyclohexylphosphine $(7.5 \mathrm{~mol} \%)$, and lutidinium chloride $(5 \mathrm{~mol} \%)$. The vessel was then placed under nitrogen. Under a positive pressure of nitrogen, $\mathrm{THF}$, the alkene ( 5 equiv), and the heterocycle $(0.1 \mathrm{M})$ were added to the vessel. The vessel was closed with the Kontes stopper and then was subjected to three successive freeze pump thaw cycles. The vessel was then heated for 15 h at $150{ }^{\circ} \mathrm{C}$. The reaction mixture was then dry loaded onto $\mathrm{SiO}_{2}$ and purifed by flash chromatography using EtOAc/hexanes.

Method C. In air to an oven dried vessel with a Kontes stopper was added $\left[\mathrm{RhCl}(\mathrm{coe})_{2}\right]_{2}(10 \mathrm{~mol} \% \mathrm{Rh})(\mathrm{coe}=$ cis-cyclooctene $)$, tricyclohexylphosphine $(15 \mathrm{~mol} \%)$, lutidinium chloride (5 mol\%), and the heterocycle. The vessel was then placed under nitrogen. Under a positive pressure of nitrogen, THF (diluted to $0.1 \mathrm{M}$ in heterocycle) and alkene ( 5 equiv) were added to the vessel. The vessel was closed with the Kontes stopper and then was subjected to three successive freeze pump thaw cycles. The vessel was then heated for $15 \mathrm{~h}$ at $150{ }^{\circ} \mathrm{C}$. The reaction mixture was then dry loaded onto $\mathrm{SiO}_{2}$ and purifed by flash chromatography using EtOAc/hexanes.

Method D. In air to an oven dried vessel with a Kontes stopper was added $\left[\mathrm{RhCl}(\mathrm{coe})_{2}\right]_{2}(10 \mathrm{~mol} \% \mathrm{Rh})(\mathrm{coe}=$ cis-cyclooctene $)$, tricyclohexylphosphine $(15 \mathrm{~mol} \%)$, and lutidinium chloride ( $5 \mathrm{~mol} \%)$. The vessel was then placed under nitrogen. Under a positive pressure of nitrogen, THF, the alkene (5 equiv), and the heterocycle $(0.1 \mathrm{M})$ were 
added to the vessel. The vessel was closed with the Kontes stopper and then was subjected to three successive freeze pump thaw cycles. The vessel was then heated for 15 h at $150{ }^{\circ} \mathrm{C}$. The reaction mixture was then dry loaded onto $\mathrm{SiO}_{2}$ and purifed by flash chromatography using EtOAc/hexanes.

2-(3, 3-Dimethyl-butyl)-1H-benzimidazole (4). Reaction of benzimidazole (154 $\mathrm{mg}, 1.31 \mathrm{mmol})$ with neo-hexene $(810 \mu \mathrm{L}, 6.30 \mathrm{mmol})$ according to method A yielded the product $(254 \mathrm{mg}, 96 \%)$ as a white solid after column chromatography (10-25\% EtOAc/hexanes). Before dry loading the crude reaction solution onto $\mathrm{SiO}_{2}$, triethylamine was added to neutralize the lutidinium chloride. The crude solution was then dry loaded on to $\mathrm{SiO}_{2}$ and purified by column chromatography. IR(KBr): 3057.2 (br, s), 1415.8, 1273.5, $736.7 \mathrm{~cm}^{-1} .{ }^{1} \mathrm{H}$ NMR $\left(\mathrm{CD}_{3} \mathrm{OD}, 500 \mathrm{MHz}\right): \delta 0.99(\mathrm{~s}, 9 \mathrm{H}), 1.8-1.65(\mathrm{~m}, 2 \mathrm{H}), 2.9-$ $2.8(\mathrm{~m}, 2 \mathrm{H}), 7.2-7.1(\mathrm{~m}, 2 \mathrm{H}), 7.5-7.4(\mathrm{~m}, 2 \mathrm{H}) .{ }^{13} \mathrm{C} \mathrm{NMR}\left(\mathrm{CD}_{3} \mathrm{OD}, 125 \mathrm{MHz}\right): \delta$ 156.1, 121.6, 41.9, 29.8, 28.0, 24.0 (Note: two carbons were not observed due the slow exchange of the N-H proton, which causes these signals to be broadened). Anal. Calcd for $\mathrm{C}_{13} \mathrm{H}_{18} \mathrm{~N}_{2}$ : C, 77.18; H, 8.97; N, 13.85. Found: C, 77.04; H, 9.06; N, 13.77. EI: Calc'd for $\mathrm{C}_{13} \mathrm{H}_{18} \mathrm{~N}_{2}(\mathrm{M}+)$ : 202. Found: 202 .

2-(3, 3-Dimethyl-butyl)-benzothiazole (5). Reaction of benzothiazole (145 $\mu \mathrm{L}, 1.28 \mathrm{mmol})$ with neo-hexene $(810 \mu \mathrm{L}, 6.30 \mathrm{mmol})$ according to method $\mathbf{B}$ yielded the product $(272 \mathrm{mg}, 97 \%)$ as a brown/orange solid after column chromatography $(2.5 \%$ EtOAc/hexanes). Before dry loading the crude reaction solution onto $\mathrm{SiO}_{2}$, triethylamine was added to neutralize the lutidinium chloride. The crude solution was then dry loaded on to $\mathrm{SiO}_{2}$ and purified by column chromatography. IR(NaCl): 2954.7, 2905.1, 2864.9, 758.4, $728.6 \mathrm{~cm}^{-1} .{ }^{1} \mathrm{H}$ NMR (500 MHz): $\delta 1.00(\mathrm{~s}, 9 \mathrm{H}), 1.9-1.7(\mathrm{~m}, 2 \mathrm{H}), 3.2-3.0(\mathrm{~m}, 2 \mathrm{H})$, 7.34 (app. triplet, $1 \mathrm{H}, J=7.5), 7.44$ (app. triplet, $1 \mathrm{H}, J=7.5), 7.83(\mathrm{~d}, 1 \mathrm{H}, J=8), 7.96$ $(\mathrm{d}, 1 \mathrm{H}, J=8) .{ }^{13} \mathrm{C} \mathrm{NMR}(125 \mathrm{MHz}): \delta 173.0,153.2,135.1,125.8,124.5,122.4,121.4$, 
43.7, 30.6, 30.1, 29.1. Anal. Calcd for $\mathrm{C}_{13} \mathrm{H}_{17} \mathrm{NS}$ : C, 71.18; H, 7.81; N, 6.39. Found: C, 71.19; H, 7.89; N, 6.52.

2-(3, 3-Dimethyl-butyl)-1H-benzoxazole (6). Reaction of benzoxazole (154 mg, $1.27 \mathrm{mmol})$ with neo-hexene $(810 \mu \mathrm{L}, 6.30 \mathrm{mmol})$ according to method $\mathbf{A}$ yielded the product (199 $\mathrm{mg}, 77 \%$ ) as a white solid after column chromatography $2.5-10 \%$ EtOAc/hexanes). Before dry loading the crude reaction solution onto $\mathrm{SiO}_{2}$, triethylamine was added to neutralize the lutidinium chloride. The crude solution was then dry loaded on to $\mathrm{SiO}_{2}$ and purified by column chromatography. IR(NaCl): 3055.5, 2955.7, 2867.1, 1572.7, 1455.3, 1242.8, $744.8 \mathrm{~cm}^{-1} .{ }^{1} \mathrm{H}$ NMR (500 MHz): $\delta 0.97(\mathrm{~s}, 9 \mathrm{H}), 1.9-1.8(\mathrm{~m}$, 2H), 3.0-2.8 (m, 2H), 7.3-7.2 (m, 2H), 7.5-7.4 (m, 1H), 7.7-7.6 (m, 1H). ${ }^{13} \mathrm{C}$ NMR (125 MHz): $\delta 167.9,150.7,141.3,124.3,124.0,119.4,110.1,40.5,30.3,29.0,24.3$. Anal. Calcd for $\mathrm{C}_{13} \mathrm{H}_{17} \mathrm{NO}$ : C, 76.81; H, 8.43; N, 6.89 Found: C, 76.42; H, 8.80; N, 6.95.

2-(3, 3-Dimethyl-butyl)-4, 5-dimethyl-thiazole (7). Reaction of 4, 5dimethylthiazole $(140 \mu \mathrm{L}, 1.32 \mathrm{mmol})$ with neo-hexene $(810 \mu \mathrm{L}, 6.30 \mathrm{mmol})$ according to method $\mathbf{B}$ yielded the product (257 $\mathrm{mg}, 99 \%$ ) as a red/orange liquid after column chromatography (5\% EtOAc/hexanes). Before dry loading the crude reaction solution onto $\mathrm{SiO}_{2}$, triethylamine was added to neutralize the lutidinium chloride. The crude solution was then dry loaded on to $\mathrm{SiO}_{2}$ and purified by column chromatography. IR(NaCl): 2954.0, 2866.1, 1477.8, 1365.0, $1121.5 \mathrm{~cm}^{-1} .{ }^{1} \mathrm{H}$ NMR (500 MHz): $\delta 0.93$ (s, $9 \mathrm{H}), 1.7-1.6(\mathrm{~m}, 2 \mathrm{H}), 2.26(\mathrm{~s}, 3 \mathrm{H}), 2.28(\mathrm{~s}, 3 \mathrm{H}), 2.9-2.8(\mathrm{~m}, 2 \mathrm{H}) .{ }^{13} \mathrm{C}$ NMR $(125 \mathrm{MHz}): \delta$ 167.5, 147.0, 124.7, 44.3, 30.5, 29.1, 29.0, 14.5, 11.2. Anal. Calcd for $\mathrm{C}_{11} \mathrm{H}_{19} \mathrm{NS}: \mathrm{C}$, 66.95; H, 9.70; N, 7.10 Found: C, 66.61; H, 9.58; N, 7.22.

8-(3, 3-Dimethyl-butyl)-7H-purine (8). Reaction of purine (152 mg, $1.27 \mathrm{mmol})$ with neo-hexene $(810 \mu \mathrm{L}, 6.30 \mathrm{mmol})$ according to method $\mathbf{A}$ (with the exception that the reaction was only heated for $4.5 \mathrm{~h}$ ) yielded the product (197 $\mathrm{mg}, 76 \%)$ as a white solid after column chromatography (50-100\% EtOAc/hexanes). IR(KBr): 2926.5 (br, s), 
1616.7, 1592.1, 920.8, 648.6, 614,1 $\mathrm{cm}^{-1} .{ }^{1} \mathrm{H}$ NMR (CD $\left.3 \mathrm{OD}, 500 \mathrm{MHz}\right): \delta 1.00(\mathrm{~s}, 9 \mathrm{H})$, 1.8-1.7 (m, 2H), 3.0-2.9 (m, 2H), $8.83(\mathrm{~s}, 1 \mathrm{H}), 8.90(\mathrm{~s}, 1 \mathrm{H}) .{ }^{13} \mathrm{C}$ NMR (125 MHz): $\delta$ 151.3, 41.3, 29.9, 27.9, 24.6 (Note: two carbons were not observed due the slow exchange of the N-H proton, which causes these signals to be broadened). Anal. Calcd for $\mathrm{C}_{11} \mathrm{H}_{16} \mathrm{~N}_{4}$ : C, 64.68; H, 7.89; N, 27.43. Found: C, 64.48; H, 7.91; N, 27.04. EI: Calc'd for $\mathrm{C}_{11} \mathrm{H}_{16} \mathrm{~N}_{4}(\mathrm{M}+)$ : 204. Found: 204.

6, 8-Bis-(3, 3-Dimethyl-butyl)-7H-purine (9). Reaction of purine (152 mg, 1.27 mmol) with neo-hexene $(810 \mu \mathrm{L}, 6.30 \mathrm{mmol})$ according to method $\mathbf{A}$ (with the exception that the reaction was only heated for $4.5 \mathrm{~h}$ ) yielded the product $(61 \mathrm{mg}, 17 \%)$ as a white solid after column chromatography (50-100\% EtOAc/hexanes). IR(KBr): 2960.2 (br, s), 1594.9, 983.4, 653.1 $\mathrm{cm}^{-1} .{ }^{1} \mathrm{H}$ NMR (400 MHz): $\delta 1.00(\mathrm{~s}, 9 \mathrm{H}), 1.02(\mathrm{~s}, 9 \mathrm{H}), 1.9-1.7(\mathrm{~m}$, 4H), 3.1-3.0 (m, 2H), 3.3-3.1 (m, 2H), $8.84(\mathrm{~s}, 1 \mathrm{H}) .{ }^{13} \mathrm{C}$ NMR $(100 \mathrm{MHz}): \delta 162.5$, $157.9,152.5,150.2,133.3,42.2,41.9,30.7,30.6,29.3,29.2,28.6,25.9$. Anal. Calcd for $\mathrm{C}_{17} \mathrm{H}_{28} \mathrm{~N}_{4}:$ C, 70.79; H, 9.78; N, 19.42. Found: C, 70.88; H, 9.86; N, 19.50.

2-(3, 3-Dimethyl-butyl)-1-methyl-benzimidazole (10). Reaction of 1methylbenzimidazole $(132 \mathrm{mg}, 1.00 \mathrm{mmol})$ with neo-hexene $(643 \mu \mathrm{L}, 5 \mathrm{mmol})$ according to method $\mathbf{C}$ yielded the product (144 $\mathrm{mg}, 67 \%$ ) as a white solid after column chromatography (10-30\% EtOAc/hexanes). Before dry loading the crude reaction solution onto $\mathrm{SiO}_{2}$, triethylamine was added to neutralize the lutidinium chloride. IR(NaCl): 2953.9, 2864.7, $745.0 \mathrm{~cm}^{-1} .{ }^{1} \mathrm{H}$ NMR (500 MHz): $\delta 1.02(\mathrm{~s}, 9 \mathrm{H}), 1.79-1.75$ $(\mathrm{m}, 2 \mathrm{H}), 2.85-2.82(\mathrm{~m}, 2 \mathrm{H}), 3.71(\mathrm{~s}, 3 \mathrm{H}), 7.30-7.20(\mathrm{~m}, 3 \mathrm{H}), 7.72-7.70(\mathrm{~m}, 1 \mathrm{H}) .{ }^{13} \mathrm{C}$ NMR (500 MHz): $\delta 155.8,142.5,135.8,121.9,121.7,119.0,108.7,41.4,30.4,29.6$, 29.1, 23.0. Anal. Calcd for $\mathrm{C}_{14} \mathrm{H}_{20} \mathrm{~N}_{2}$ : C, 77.73; H, 9.32; N, 12.95. Found: C, 77.39; H, $9.54 ; \mathrm{N}, 12.63$.

2-Hexyl-1H-benzimidazole (11). Reaction of benzimidazole (151 mg, 1.28 mmol) with 1-hexene (538 mg, $6.40 \mathrm{mmol}$ ) according to method A yielded the product 
(209 mg, 80\%) as a white solid after column chromatography (20-50\% EtOAc/hexanes). IR(KBr): 2930.1 (br, s), 1420.7, 1027.0, $750.2 \mathrm{~cm}^{-1} .{ }^{1} \mathrm{H}$ NMR $\left(\mathrm{CD}_{3} \mathrm{OD}, 500 \mathrm{MHz}\right): \delta$ $0.87(\mathrm{t}, 3 \mathrm{H}, J=6.5), 1.4-1.2(\mathrm{~m}, 6 \mathrm{H}), 1.85-1.75(\mathrm{~m}, 2 \mathrm{H}), 2.85(\mathrm{t}, 2 \mathrm{H}, J=7.5), 7.19-7.13$ $(\mathrm{m}, 2 \mathrm{H})$, 7.5-7.45 (m, 2H). ${ }^{13} \mathrm{C}$ NMR ( $\left.\mathrm{CD}_{3} \mathrm{OD}, 125 \mathrm{MHz}\right): \delta 155.5,121.6,31.2,28.5$, 28.3, 27.9, 22.1, 12.9 (Note: two carbons were not observed due the slow exchange of the $\mathrm{N}-\mathrm{H}$ proton, which causes these signals to be broadened). Anal. Calcd for $\mathrm{C}_{13} \mathrm{H}_{18} \mathrm{~N}_{2}$ : C, 77.18; H, 8.97; N, 13.85. Found: C, 77.36; H, 9.03; N, 13.68. EI: Calc'd for $\mathrm{C}_{13} \mathrm{H}_{18} \mathrm{~N}_{2}$ (M+): 202. Found: 202.

2-[6-(tert-Butyl-diphenyl-silanyloxy)-hexyl]-1H-benzimidazole (12). Reaction of benzimidazole (70 mg, $0.591 \mathrm{mmol})$ with tert-butyl-hex-5-enyloxy-diphenyl-silane (1 g, $2.95 \mathrm{mmol}$ ) according to method A yielded the product (207 $\mathrm{mg}, 77 \%$ ) as a white solid after column chromatography (25-50\% EtOAc/hexanes). IR(KBr): 2926.5 (br, s), 1426.2, 1273.8, 1109.3, 744.4, 701.0 $\mathrm{cm}^{-1} .{ }^{1} \mathrm{H} \mathrm{NMR}\left(\mathrm{CD}_{3} \mathrm{OD}, 500 \mathrm{MHz}\right): \delta 0.99(\mathrm{~s}, 9 \mathrm{H}), 1.36-$ $1.30(\mathrm{~m}, 2 \mathrm{H}), 1.44-1.38(\mathrm{~m}, 2 \mathrm{H}), 1.56-1.51(\mathrm{~m}, 2 \mathrm{H}), 1.82-1.77(\mathrm{~m}, 2 \mathrm{H}), 2.84(\mathrm{t}, 2 \mathrm{H}, J=$ 7.5), $3.62(\mathrm{t}, 2 \mathrm{H}, J=6.5), 7.2-7.15(\mathrm{~m}, 2 \mathrm{H}), 7.4-7.3(\mathrm{~m}, 6 \mathrm{H}), 7.5-7.45(\mathrm{~m}, 2 \mathrm{H}), 7.7-7.6(\mathrm{~m}$, 4H). ${ }^{13} \mathrm{C}$ NMR $\left(\mathrm{CD}_{3} \mathrm{OD}, 125 \mathrm{MHz}\right): \delta 155.4,135.2,133.6,129.3,127.3,121.7,63.4$, 32.0, 28.5, 28.3, 27.8, 25.9, 25.1, 18.5 (Note: two carbons were not observed due the slow exchange of the N-H proton, which causes these signals to be broadened). Anal. Calcd for $\mathrm{C}_{29} \mathrm{H}_{36} \mathrm{~N}_{2} \mathrm{OSi}$ : C, 76.27; H, 7.95; N, 6.13. Found: C, 76.16; H, 8.10; N, 6.06. FAB: Calc'd for $\mathrm{C}_{29} \mathrm{H}_{36} \mathrm{~N}_{2} \mathrm{OSi}(\mathrm{M}+1)$ : 457. Found: 457 .

4-(1H-Benzimidazol-2-yl)-butyric acid isobutyl ester (13). Reaction of benzimidazole (150 mg, $1.27 \mathrm{mmol})$ with isobutyl vinylacetate (903 mg, $6.35 \mathrm{mmol})$ according to method $\mathbf{C}$ (with the exception that the reaction was heated for only $8 \mathrm{~h}$ ) yielded the product $(288 \mathrm{mg}, 87 \%)$ as a white solid after column chromatography (4075\% EtOAc/hexanes). IR(KBr): 2947.8 (br, s), 1732.9 9(s), $749.3 \mathrm{~cm}^{-1} .{ }^{1} \mathrm{H}$ NMR $\left(\mathrm{CD}_{3} \mathrm{OD}, 500 \mathrm{MHz}\right): \delta 0.89(\mathrm{~d}, 6 \mathrm{H}, J=6.5), 1.90-1.82(\mathrm{~m}, 1 \mathrm{H}), 2.15-2.10(\mathrm{~m}, 2 \mathrm{H}), 2.41$ 
$(\mathrm{t}, 2 \mathrm{H}, J=7.5), 2.93(\mathrm{t}, 2 \mathrm{H}, J=7.5), 3.80(\mathrm{~d}, 2 \mathrm{H}, J=6.5), 7.18-7.16(\mathrm{~m}, 2 \mathrm{H}), 7.49-7.46(\mathrm{~m}$, 2H). ${ }^{13} \mathrm{C} \mathrm{NMR}\left(\mathrm{CD}_{3} \mathrm{OD}, 125 \mathrm{MHz}\right): \delta 173.2,154.4,138.0$ (br), 121.8, 113.9 (br), 70.2, 32.8, 27.6, 27.5, 23.0, 17.9. Anal. Calcd for $\mathrm{C}_{15} \mathrm{H}_{20} \mathrm{~N}_{2} \mathrm{O}_{2}: \mathrm{C}, 69.20 ; \mathrm{H}, 7.74 ; \mathrm{N}, 10.76$. Found: C, 69.14; H, 7.86; N, 10.73. EI: Calc'd for $\mathrm{C}_{15} \mathrm{H}_{20} \mathrm{~N}_{2} \mathrm{O}_{2}(\mathrm{M}+)$ : 260. Found: 260. 2-[2-(4, 4, 5, 5-Tetramethyl-[1, 3]dioxolan-2-yl)-ethyl]-1H-benzimidazole (14). Reaction of benzimidazole (150 mg, $1.27 \mathrm{mmol})$ with 4, 4, 5, 5-tetramethyl-2-vinyl-[1, 3]dioxolane (992 mg, $6.35 \mathrm{mmol}$ ) according to method $\mathbf{C}$ yielded the product (209 $\mathrm{mg}$, $60 \%$ ) as a white solid after column chromatography (25-75\% EtOAc/hexanes). Before dry loading the crude reaction solutin onto $\mathrm{SiO}_{2}$, triethylamine was added to neutralize the lutidinium chloride. The crude solution was then dry loaded on to $\mathrm{SiO}_{2}$ and purified by column chromatography. IR(KBr): 2976.2 (br, s), 1547.7, 1164.3, 1131.4, 758.9 cm². ${ }^{1} \mathrm{H}$ NMR (DMSO-d 6 , $\left.500 \mathrm{MHz}\right): \delta 1.12(\mathrm{~s}, 6 \mathrm{H}), 1.14(\mathrm{~s}, 6 \mathrm{H}), 2.01-1.97$ (m, 2H), 2.85 (t, $2 \mathrm{H}, J=8), 5.04(\mathrm{t}, 1 \mathrm{H}, J=5), 7.09-7.08(\mathrm{~m}, 2 \mathrm{H}), 7.47-7.40(\mathrm{~m}, 2 \mathrm{H}), 12.20-12.05(\mathrm{~m}, 1 \mathrm{H})$. ${ }^{13}$ C NMR (DMSO-d 6 , 125 MHz): $\delta 154.8,121.5$ (br), 99.6, 81.8, 34.4, 24.5, 24.0, 22.3 (Note: two carbons were not observed due the slow exchange of the N-H proton, which causes these signals to be broadened). Anal. Calcd for $\mathrm{C}_{16} \mathrm{H}_{22} \mathrm{~N}_{2} \mathrm{O}_{2}: \mathrm{C}, 70.04 ; \mathrm{H}, 8.08 ; \mathrm{N}$, 10.21. Found: C, 69.99; H, 8.33; N, 10.06. FAB: Calc'd for $\mathrm{C}_{16} \mathrm{H}_{22} \mathrm{~N}_{2} \mathrm{O}_{2}(\mathrm{M}+1): 275$. Found: 275.

2-Phenethyl-1H-benzimidazole (15). Reaction of benzimidazole (100 mg, 0.847 mmol) with styrene (491 $\mathrm{uL}, 4.24 \mathrm{mmol}$ ) according to method $\mathbf{C}$ yielded the product (36 mg, 19\%) as a white solid after column chromatography (25-50\% EtOAc/hexanes). The THF used in this reaction was dried over $4 \AA$ molecular sieves instead of being distilled from $\mathrm{Na}^{\circ}$ /benzophenone. GCMS analysis of the crude reaction mixture showed a linear:branched ratio of 25:1. IR(KBr): $2866.8(\mathrm{br}, \mathrm{s}), 1420.2,1280.1,749.3,696.2 \mathrm{~cm}^{-1}$. ${ }^{1} \mathrm{H}$ NMR $\left(\mathrm{CD}_{3} \mathrm{OD}, 500 \mathrm{MHz}\right): \delta 3.2-3.05(\mathrm{~m}, 4 \mathrm{H}), 7.3-7.1(\mathrm{~m}, 7 \mathrm{H}), 7.5-7.43(\mathrm{~m}, 2 \mathrm{H}) .{ }^{13} \mathrm{C}$ NMR (CD $\left.\mathrm{CD}_{3}, 125 \mathrm{MHz}\right): \delta 154.5,140.3,128.1,127.9,125.9,121.7,34.0,30.5$ (Note: 
two carbons were not observed due the slow exchange of the $\mathrm{N}-\mathrm{H}$ proton, which causes these signals to be broadened). HRMS: Calc'd for $\mathrm{C}_{15} \mathrm{H}_{14} \mathrm{~N}_{2}(\mathrm{M}+)$ : 222.1158. Found: 222.1157.

3-(4, 5-Dimethyl-thiazol-2-yl)-propionic acid tert-butyl ester (16). Reaction of 4, 5-dimethylthiazole (97\% pure, $135 \mu \mathrm{L}, 1.24 \mathrm{mmol})$ with tert-butyl acrylate $(930 \mu \mathrm{L}$, $6.35 \mathrm{mmol})$ according to method $\mathbf{D}$ yielded the product $(278 \mathrm{mg}, 93 \%)$ as a red-orange liquid after column chromatography (5-10\% EtOAc/hexanes). Before dry loading the crude reaction onto $\mathrm{SiO}_{2}$, triethylamine was added to neturalize the lutidinium chloride. The crude solution was then dry loaded on to $\mathrm{SiO}_{2}$ and purified by column chromatography. IR(NaCl): 2978.9, 2924.5, 1729.1, 1366.88, $1154.65 \mathrm{~cm}^{-1} .{ }^{1} \mathrm{H}$ NMR $(500 \mathrm{MHz}): \delta 1.42(\mathrm{~s}, 9 \mathrm{H}), 2.25(\mathrm{~s}, 3 \mathrm{H}), 2.29(\mathrm{~s}, 3 \mathrm{H}), 2.66(\mathrm{t}, 2 \mathrm{H}, J=7.5), 3.14(\mathrm{t}, 2 \mathrm{H}, J=$ 7.5). ${ }^{13} \mathrm{C}$ NMR (125 MHz): $\delta 171.4,164.3,147.3,125.2,80.6,35.1,28.5,28.0,14.5$, 11.2. Anal. Calcd for $\mathrm{C}_{12} \mathrm{H}_{19} \mathrm{NO}_{2} \mathrm{~S}$ : C, 59.72; H, 7.93; N, 5.80. Found: C, 59.75; H, 7.79; N, 6.00 .

3-(4, 5-Dimethyl-thiazol-2-yl)-propionitrile(17). Reaction of 4, 5dimethylthiazole (97\% pure, $135 \mu \mathrm{L}, 1.24 \mathrm{mmol})$ with acrylonitrile $(418 \mu \mathrm{L}, 6.35 \mathrm{mmol})$ according to method $\mathbf{D}$ yielded the product (134 $\mathrm{mg}, 65 \%$ linear product) as a yellow liquid after column chromatography (10-30\% EtOAc/hexanes). GCMS analysis of the crude reaction mixture showed a linear:branched ratio of 4.3:1. IR(NaCl): 2922.8, 2864.8, 2249.3, 1439.7, $1134.8 \mathrm{~cm}^{-1} .{ }^{1} \mathrm{H}$ NMR (400 MHz): $\delta 2.27$ (s, 3H), $2.30(\mathrm{~s}, 3 \mathrm{H})$, $2.80(\mathrm{t}, 2 \mathrm{H}, J=7.6), 3.19(\mathrm{t}, 2 \mathrm{H}, J=7.6) .{ }^{13} \mathrm{C}$ NMR (100 MHz): $\delta 160.6,148.2,126.5$, 118.6, 28.9, 17.3, 14.6, 11.2. Anal. Calcd for $\mathrm{C}_{8} \mathrm{H}_{10} \mathrm{~N}_{2} \mathrm{~S}: \mathrm{C}, 57.80 ; \mathrm{H}, 6.06 ; \mathrm{N}, 16.85$. Found: C, 57.81; H, 6.05; N, 17.04. 


\section{Additive Table}

\section{(a) General Procedure for Additive Screen}

In the glove box, to a vial was added compound $1(0.15 \mathrm{M}), \mathrm{PCy}_{3}(0.023 \mathrm{M})$, $\left[\mathrm{RhCl}(\mathrm{coe})_{2}\right]_{2}(0.015 \mathrm{M}$ in $\mathrm{Rh})$, and dimethoxytoluene (internal standard) in THF. The solution $(0.5 \mathrm{~mL})$ was used to transfer the additive $(10 \mathrm{~mol} \%)$ into a vessel with a Kontes top. If the additive was not soluble then the solution in the reaction vessel was decanted off and was used to transfer the remaining additive into the vessel. This procedure was repeated until the additive was transferred. The reaction mixture was then heated to 105 ${ }^{\circ} \mathrm{C}$ for 15 to $17 \mathrm{~h}$. The reaction mixture was cooled to $\mathrm{rt}$ and then the solution was concentrated. The residue was then dissolved in $\mathrm{CDCl}_{3}$ and analyzed by ${ }^{1} \mathrm{H} \mathrm{NMR}$ spectroscopy.

(b) Table S-1. Complete Additive Table

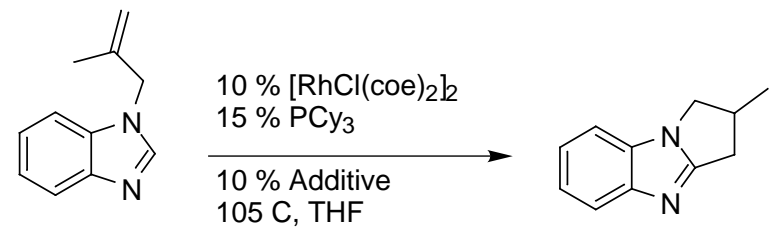

\begin{tabular}{cccc}
\hline Additive 10\% & $\%$ SM & $\%$ Product & Time \\
\hline None & 77 & $<7$ & $15 \mathrm{~h}$ \\
Benzoic Acid & 67 & 10 & $16 \mathrm{~h}$ \\
$\mathrm{TfOH}$ & Decomp & Decomp & $16 \mathrm{~h}$ \\
$\mathrm{NH}_{4} \mathrm{Cl}$ & 66 & 11 & $16 \mathrm{~h}$ \\
$\mathrm{H}_{2} \mathrm{O}$ pTSA & 57 & 22 & $16 \mathrm{~h}$ \\
Pyridinium HCl & 75 & 25 & $16 \mathrm{~h}$ \\
Pyridinium HBr & 64 & 35 & $16 \mathrm{~h}$ \\
Lutidinium HI & 53 & 39 & $15 \mathrm{~h}$ \\
Lutidinium HBr & 54 & 42 & $15 \mathrm{~h}$ \\
Lutidinium HCL & 32 & 49 & $17 \mathrm{~h}$ \\
Lutidinium pTSA & 50 & 28 & $16 \mathrm{~h}$ \\
$\mathrm{Bu}_{4} \mathrm{NCl}$ & 70 & 8 & $15 \mathrm{~h}$ \\
$\mathrm{PPNCl}_{\mathrm{Cu}(\mathrm{OTf})_{2}}$ & 71 & 9 & $15 \mathrm{~h}$ \\
& Decomp & None & $15 \mathrm{~h}$ \\
\hline
\end{tabular}




\begin{tabular}{cccc}
\hline $\mathrm{Cu}(\mathrm{OTf})_{2} \mathrm{PheBox}$ (in toluene) & Decomp & None & $17 \mathrm{~h}$ \\
$\mathrm{CuCl}_{2}$ & 72 & 6 & $15 \mathrm{~h}$ \\
$\mathrm{ZnCl}_{2}$ & 72 & 12 & $15 \mathrm{~h}$ \\
$\mathrm{Al}(\mathrm{OTf})_{3}$ & 76 & Trace & $15 \mathrm{~h}$ \\
$\mathrm{ScCl}_{3}$ & 44 & 33 & $15 \mathrm{~h}$ \\
$\mathrm{Sc}(\mathrm{OTf})_{3}$ & 85 & 14 & $17 \mathrm{~h}$ \\
$\mathrm{YCl}_{3}$ & 19 & 69 & $16 \mathrm{~h}$ \\
$\mathrm{YBr}_{3}$ & 47 & 34 & $16 \mathrm{~h}$ \\
$\mathrm{Mg}\left(\mathrm{OTf}_{2}\right.$ & 85 & 14 & $16 \mathrm{~h}$ \\
$\mathrm{MgBr}_{2}$ & 12 & 69 & $16 \mathrm{~h}$ \\
$\mathrm{MgCl}_{2}$ & 47 & 27 & $15 \mathrm{~h}$ \\
$\mathrm{MgF}_{2}$ & 72 & - & $17 \mathrm{~h}$ \\
$\mathrm{MgI}_{2}$ & 50 & 18 & $17 \mathrm{~h}$ \\
$\mathrm{LiBr}_{\mathrm{NaBr}}$ & 58 & 24 & $16 \mathrm{~h}$ \\
$\mathrm{KBr}_{\mathrm{CaCl}}$ & 77 & Trace & $16 \mathrm{~h}$ \\
& 78 & Trace & $16 \mathrm{~h}$ \\
& 68 & 13 & $16 \mathrm{~h}$ \\
\hline
\end{tabular}

Run at $0.15 \mathrm{M}$ substrate; yields by NMR

\section{Structrual Data on 9}

\section{Figure S-1-ORTEP of 9}

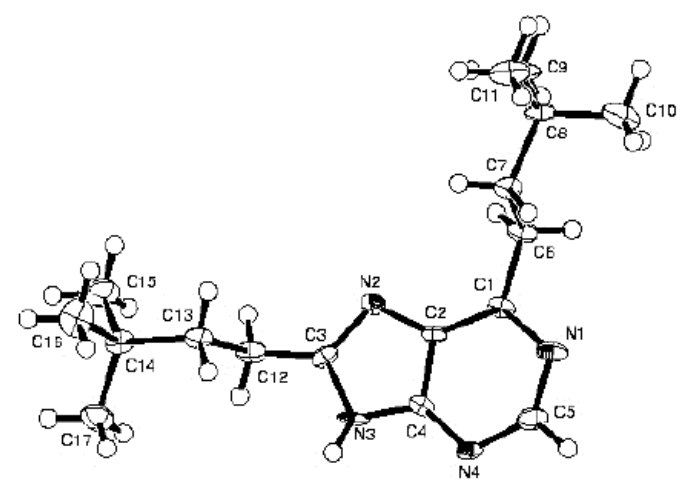

\footnotetext{
${ }^{i}$ Tan, K. L.; Bergman, R. G.; Ellman, J. A. J. Am. Chem. Soc. 2001, 123, 2685-2686.

ii Inorg. Syth., 1973, 14, 92-93.

iii Fujiwara, K.; Amano, A.; Tokiwano, T.; Murai, A. Tetrahedron 2000, 56, 1065-1080.

${ }^{\text {iv }}$ Deagostino, A.; Maddaluno, J.; Mella, M.; Prandi, C.; Venturello, P. J. Chem. Soc.-Perkin Trans. 1 1998, 881-888.
} 